\title{
On the Symmetrical System of Rational Difference Equation $x_{n+1}=A+y_{n-k} / y_{n}, y_{n+1}=A+x_{n-k} / x_{n}{ }^{*}$
}

\author{
Decun Zhang, Wenqiang Jï, Liying Wang, Xiaobao Li \\ Institute of Systems Science and Mathematics, Naval Aeronautical and Astronautical University, Yantai, China \\ Email: \#wqyikeshu@163.com
}

Received March 12, 2013; revised April 12, 2013; accepted April 19, 2013

Copyright (C) 2013 Decun Zhang et al. This is an open access article distributed under the Creative Commons Attribution License, which permits unrestricted use, distribution, and reproduction in any medium, provided the original work is properly cited.

\section{ABSTRACT}

In this paper, we study the behavior of the symmetrical system of rational difference equation:

$$
x_{n+1}=A+\frac{y_{n-k}}{y_{n}}, y_{n+1}=A+\frac{x_{n-k}}{x_{n}}, n=0,1, \cdots
$$

where $A>0$ and $x_{i}, y_{i} \in(0, \infty)$, for $i=-k,-k+1, \cdots, 0$.

Keywords: Symmetrical System; Difference Equation; Boundedness; Period-Two Solution

\section{Introduction}

Recently there has been a great interest in studying difference equations and systems, and quite a lot of papers about the behavior of positive solutions of system of difference equation. We can read references [1-10].

In [1] C. Cina studied the system:

$$
x_{n+1}=\frac{1}{y_{n}}, y_{n+1}=\frac{y_{n}}{x_{n-1} y_{n-1}}, n=0,1, \cdots .
$$

In [2] A. Y. Ozban studied the difference equation system:

$$
x_{n+1}=\frac{1}{y_{n-k}}, y_{n+1}=\frac{y_{n}}{x_{n-m} y_{n-m-k}}, n=0,1, \cdots .
$$

In [3] A. Y. Ozban studied the behavior of positive solutions of the difference equation system:

$$
x_{n}=\frac{a}{y_{n-3}}, y_{n}=\frac{b y_{n-3}}{x_{n-q} y_{n-q}}, n=0,1, \cdots .
$$

In [4] X. Yang, Y. Liu, S. Bai studied the difference equation system:

$$
x_{n+1}=\frac{a}{y_{n-p}}, y_{n+1}=\frac{b y_{n-p}}{x_{n-q} y_{n-q}}, n=0,1, \cdots .
$$

\footnotetext{
"Research supported by Distinguished Expert Foundation and Youth Science Foundation of Naval Aeronautical and Astronautical University.

"Corresponding author.
}

We can see in [1-4], they have the same similar character, which is the system can be reduced into a difference equation with $x_{n}$ or $y_{n}$.

In [5] G. Papaschinopoulos, C. J. Schinas studied the behavior of positive solutions of the difference equation system:

$$
x_{n+1}=A+\frac{y_{n}}{x_{n-p}}, y_{n+1}=A+\frac{x_{n}}{y_{n-p}}, n=0,1, \cdots .
$$

In [6] G. Papaschinopoulos, Basil K. Papadopoulos studied the behavior of positive solutions of the difference equation system:

$$
x_{n+1}=A+\frac{x_{n}}{y_{n-p}}, y_{n+1}=B+\frac{y_{n}}{x_{n-p}}, n=0,1, \cdots .
$$

In [7] E. Camouzis, G. Papaschinopoulos studied the behavior of positive solutions of the difference equation system:

$$
x_{n+1}=1+\frac{x_{n}}{y_{n-m}}, y_{n+1}=1+\frac{y_{n}}{x_{n-m}}, n=0,1, \cdots .
$$

In [8] Yu Zhang, Xiaofan Yang, David J. Evans, Ce Zhu studied the behavior of positive solutions of the difference equation system:

$$
x_{n+1}=A+\frac{y_{n-m}}{x_{n}}, y_{n+1}=A+\frac{x_{n-m}}{y_{n}}, n=0,1, \cdots .
$$

Motivated by systems above, we introduce the sym- 
metrical system:

$$
x_{n+1}=A+\frac{y_{n-k}}{y_{n}}, y_{n+1}=A+\frac{x_{n-k}}{x_{n}}, n=0,1, \cdots .
$$

with parameter $A>0$, the initial conditions $x_{i}, y_{i}>0$, for $i=-k,-k+1, \cdots, 0$, and $k$ is a positive integer. We can easily get the system (9) has the unique positive equilibrium $(\bar{x}, \bar{y})=(A+1, A+1)$.

There are two cases we need to consider:

1) If the initial conditions $x_{i}=y_{i}$ in the system (9) for $i=-k,-k+1, \cdots, 0$, then $x_{n}=y_{n}$ for all $n \geq-k$, thus, the system (9) reduces to the difference equation

$$
x_{n+1}=A+\frac{x_{n-k}}{x_{n}}
$$

which was studied by El-owaidy in [11].

2) If $x_{i} \neq y_{i}$ for $i \in\{-k,-k+1, \cdots, 0\}$, then the system (9) is similar to the system in [8]. We study the system (9) basing on this condition in this paper.

In this paper, we try to give some results of the system (9) by using the methods in [8]. We consider the following cases of $0<A<1, A=1$ and $A>1$.

\section{The Case $0<A<1$}

In this section, we give the asymptotic behavior of positive solution to the system (9).

Theorem 2.1. Suppose $0<A<1$ and $\left\{x_{n}, y_{n}\right\}$ is an arbitrary positive solution of the system (9). Then the following statements hold.

1) If $k$ is odd, and $0<x_{2 m-1}<1,0<y_{2 m-1}<1$,

$x_{2 m}>\frac{1}{1-A}, \quad y_{2 m}>\frac{1}{1-A}$ for $m=\frac{1-k}{2}, \frac{3-k}{2}, \cdots, 0$, then

$$
\lim _{n \rightarrow \infty} x_{2 n}=\infty, \lim _{n \rightarrow \infty} y_{2 n}=\infty, \lim _{n \rightarrow \infty} x_{2 n+1}=A, \lim _{n \rightarrow \infty} y_{2 n+1}=A .
$$

2) If $k$ is odd, and $0<x_{2 m}<1,0<y_{2 m}<1$,

$x_{2 m-1}>\frac{1}{1-A}, \quad y_{2 m-1}>\frac{1}{1-A}$ for $m=\frac{1-k}{2}, \frac{3-k}{2}, \cdots, 0$, then

$$
\lim _{n \rightarrow \infty} x_{2 n}=A, \lim _{n \rightarrow \infty} y_{2 n}=A, \lim _{n \rightarrow \infty} x_{2 n+1}=\infty, \lim _{n \rightarrow \infty} y_{2 n+1}=\infty .
$$

3) If $k$ is even, we can not get some useful results.

Proof: 1) Obviously, we can have

$$
\begin{gathered}
0<x_{1}=A+\frac{y_{-k}}{y_{0}}<A+\frac{1}{y_{0}}<A+(1-A)=1, \\
0<y_{1}=A+\frac{x_{-k}}{x_{0}}<A+\frac{1}{x_{0}}<A+(1-A)=1, \\
x_{2}=A+\frac{y_{1-k}}{y_{1}}>A+y_{1-k}>y_{1-k}>\frac{1}{1-A},
\end{gathered}
$$

$$
y_{2}=A+\frac{x_{1-k}}{x_{1}}>A+x_{1-k}>x_{1-k}>\frac{1}{1-A} .
$$

By introduction, we can get

$$
0<x_{2 n+1}<1,0<y_{2 n+1}<1, x_{2 n}>\frac{1}{1-A}, y_{2 n}>\frac{1}{1-A},
$$

for $n=0,1,2, \cdots$.

So for $n \geq \frac{k+2}{2}$,

$$
\begin{aligned}
x_{2 n} & =A+\frac{y_{2 n-(k+1)}}{y_{2 n-1}}>A+y_{2 n-(k+1)} \\
& =2 A+\frac{x_{2 n-(2 k+2)}}{x_{2 n-k-2}}>2 A+x_{2 n-(2 k+2)} .
\end{aligned}
$$

By limiting the inequality above, we can get $\lim _{n \rightarrow \infty} x_{2 n}=+\infty$. Similarly, we can also get $\lim _{n \rightarrow \infty} y_{2 n}=+\infty$.

Taking limits on the both sides of the following two equations

$$
x_{2 n+1}=A+\frac{y_{2 n-k}}{y_{2 n}}, y_{2 n+1}=A+\frac{x_{2 n-k}}{x_{2 n}}
$$

we can obtain $\lim _{n \rightarrow \infty} x_{2 n+1}=A, \quad \lim _{n \rightarrow \infty} y_{2 n+1}=A$.

The proof of 2) is similar, so we omit it.

\section{The Case $A=1$}

In this section, we try to get the boundedness, persistence, and periodicity of positive solutions of the system (9).

Theorem 3.1. Suppose $A=1$. Then every positive solution of the system (9) is bounded and persists.

Proof. $\left\{x_{n}, y_{n}\right\}_{n=-k}^{\infty}$ is a positive solution of the system (9).

Obviously, $x_{n}>1, y_{n}>1$, for $n \geq 1$. So we can get

$$
x_{i}, y_{i} \in\left[L, \frac{L}{L-1}\right], i=1,2, \cdots, k+1 \text {, }
$$

where $L=\min \left\{a, \frac{b}{b-1}\right\}>1, \quad a=\min \left\{x_{i}, y_{i}\right\}$, $b=\max \left\{x_{i}, y_{i}\right\}$, for $1 \leq i \leq k+1$.

Then we can obtain

$$
\begin{aligned}
L & =1+\frac{L}{L /(L-1)} \leq x_{k+2} \\
& =1+\frac{y_{1}}{y_{k+1}} \leq 1+\frac{L /(L-1)}{L}=\frac{L}{L-1} \\
L & =1+\frac{L}{L /(L-1)} \leq y_{k+2} \\
& =1+\frac{x_{1}}{x_{k+1}} \leq 1+\frac{L /(L-1)}{L}=\frac{L}{L-1}
\end{aligned}
$$


By introduction, we have

$$
x_{i}, y_{i} \in\left[L, \frac{L}{L-1}\right], i=1,2, \cdots .
$$

Hence, we complete the proof.

Theorem 3.2. Suppose $A=1,\left\{x_{n}, y_{n}\right\}_{n=-k}^{\infty}$ is a positive solution of the system (9). Then

$$
\begin{aligned}
& \liminf _{n \rightarrow \infty} x_{n}=\liminf _{n \rightarrow \infty} y_{n}, \\
& \lim _{n \rightarrow \infty} \sup x_{n}=\lim _{n \rightarrow \infty} \sup y_{n} .
\end{aligned}
$$

Proof: By (10), we can get

$$
\begin{gathered}
l_{1}=\liminf _{n \rightarrow \infty} x_{n} \geq L>1, \\
l_{2}=\lim _{n \rightarrow \infty} \inf y_{n} \geq L>1 . \\
U_{1}=\lim _{n \rightarrow \infty} \sup x_{n}>1, \\
U_{2}=\lim _{n \rightarrow \infty} \sup y_{n}>1 .
\end{gathered}
$$

By system (9), we can have

$$
U_{1} \leq 1+\frac{U_{2}}{l_{2}}, U_{2} \leq 1+\frac{U_{1}}{l_{1}}, l_{1} \geq 1+\frac{l_{2}}{U_{2}}, l_{2} \geq 1+\frac{l_{1}}{U_{1}}
$$

which implies $U_{1} l_{2} \leq l_{2}+U_{2} \leq l_{1} U_{2} \leq l_{1}+U_{1} \leq l_{2} U_{1}$.

Hence, we can obtain

$$
l_{1}+U_{1}=l_{2}+U_{2}, l_{1} U_{2}=l_{2} U_{1},
$$

which can be changed into

$$
l_{1}+\left(-U_{2}\right)=l_{2}+\left(-U_{1}\right), l_{1}\left(-U_{2}\right)=l_{2}\left(-U_{1}\right) .
$$

Obviously, $l_{1}=l_{2}, U_{1}=U_{2}$, we complete the proof.

Theorem 3.3. Suppose $A=1$.

1) If $k$ is odd, then every positive solution of the system (9) with prime period two takes the form

$$
(a, a),\left(\frac{a}{a-1}, \frac{a}{a-1}\right),(a, a),\left(\frac{a}{a-1}, \frac{a}{a-1}\right), \cdots .
$$

or

$$
\left(a, \frac{a}{a-1}\right),\left(\frac{a}{a-1}, a\right),\left(a, \frac{a}{a-1}\right),\left(\frac{a}{a-1}, a\right), \cdots .
$$

with $1<a \neq 2$.

2) If $m$ is even, there do not exist positive nontrival solution of the system (9) with prime period two.

Proof: 1) As $k$ is odd.

We set $\left\{x_{n}, y_{n}\right\}$ is the solution of the system (9) with prime period two. Then there are four positive number $A, B, C, D>1$ such that

$$
x_{2 n-k}=A, y_{2 n-k}=B, x_{2 n+1-k}=C, y_{2 n+1-k}=D, n=0,1, \cdots \text {. }
$$

If $A=C$, by the system (9) we can get $B=D=2$, which is contradiction with the condition $a \neq 2$, hence $A \neq C$. Similarly, we can get $B \neq D$. Then we obtain

$$
\begin{aligned}
& \liminf _{n \rightarrow \infty} x_{n}=\min \{A, C\}, \\
& \liminf _{n \rightarrow \infty} y_{n}=\min \{B, D\} . \\
& \limsup _{n \rightarrow \infty} x_{n}=\max \{A, C\}, \\
& \lim _{n \rightarrow \infty} \sup y_{n}=\max \{B, D\} .
\end{aligned}
$$

From Theorem 3.2, we can get

$$
\begin{aligned}
& \min \{A, C\}=\min \{B, D\} \\
& \max \{A, C\}=\max \{B, D\}
\end{aligned}
$$

Next, we consider the following possibilities:

Case 1: Either(I) $A<C$ and $B<D$ or (II) $A>C$ and $B$ $>D$. Then $A=B, C=D$.

Case 2: Either(I) $A<C$ and $B>D$ or (II) $A>C$ and $B$ $<D$. Then $A=D, B=C$.

Therefore by the system (9), we can get 1 ) holds.

2) Obviously, if $k$ is even, the system (9) just has trival solution with prime period two.

We complete the proof.

\section{The Case $A>1$}

Theorem 4.1. Suppose $A>1$. Then every positive solution of the system (9) is bounded and persists.

Proof. Let $\left\{x_{n}, y_{n}\right\}$ be a positive solution of the system (9). Obviously, $x_{n}>A>1, y_{n}>A>1$, for $n \geq 1$. So we can get

$$
x_{i}, y_{i} \in\left[L, \frac{L}{L-A}\right], i=1,2, \cdots, k+1,
$$

where $L=\min \left\{a, \frac{b}{b-A}\right\}>1, a=\min \left\{x_{i}, y_{i}\right\}$, $b=\max \left\{x_{i}, y_{i}\right\}$, for $1 \leq i \leq k+1$. Then we can obtain

$$
\begin{aligned}
L & =A+\frac{L}{L /(L-A)} \leq x_{k+2} \\
& =A+\frac{y_{1}}{y_{k+1}} \leq A+\frac{L /(L-A)}{L}=\frac{L}{L-A} \\
L & =A+\frac{L}{L /(L-A)} \leq y_{k+2} \\
& =A+\frac{x_{1}}{x_{k+1}} \leq A+\frac{L /(L-A)}{L}=\frac{L}{L-A}
\end{aligned}
$$

By introduction, we have

$$
x_{i}, y_{i} \in\left[L, \frac{L}{L-A}\right], i=1,2, \cdots .
$$

We complete the proof.

Theorem 4.2. Suppose $A>1$. Then every positive solution of the system (9) converges to the equilibrium as $n \rightarrow \infty$. 
Proof: By (13), we can get

$$
\begin{gathered}
l_{1}=\liminf _{n \rightarrow \infty} x_{n} \geq L>A>1, \\
l_{2}=\liminf _{n \rightarrow \infty} y_{n} \geq L>A>1 . \\
U_{1}=\lim _{n \rightarrow \infty} \sup x_{n}>A>1, \\
U_{2}=\lim _{n \rightarrow \infty} \sup y_{n}>A>1 .
\end{gathered}
$$

By system (9), we can have

$$
U_{1} \leq A+\frac{U_{2}}{l_{2}}, U_{2} \leq A+\frac{U_{1}}{l_{1}}, l_{1} \geq A+\frac{l_{2}}{U_{2}}, l_{2} \geq A+\frac{l_{1}}{U_{1}}
$$

which imply

$$
\begin{gathered}
A U_{1}+l_{1} \leq U_{1} l_{2} \leq A l_{2}+U_{2}, \\
A U_{2}+l_{2} \leq U_{2} l_{1} \leq A l_{1}+U_{1}, \\
l_{1}+A U_{1}-\left(A l_{1}+U_{1}\right) \leq A l_{2}+U_{2}-\left(l_{2}+A U_{2}\right), \\
(A-1)\left(U_{1}-l_{1}+U_{2}-l_{2}\right) \leq 0 .
\end{gathered}
$$

By the condition $A>1$, we can get

$$
U_{1}-l_{1}+U_{2}-l_{2}=0
$$

Besides, $U_{1}-l_{1} \geq 0$ and $U_{2}-l_{2} \geq 0$, so we can get $U_{1}-l_{1}=0$ and $U_{2}-l_{2}=0$.

i.e.

$$
U_{1}=l_{1}, U_{2}=l_{2}
$$

we complete the proof.

\section{REFERENCES}

[1] C. Cinar, "On the Positive Solutions of the Difference Equation System $x_{n+1}=\frac{1}{y_{n}}, \quad y_{n+1}=\frac{y_{n}}{x_{n-1} y_{n-1}}$," Applied Mathematics and Computation, Vol. 158, No. 2, 2004, pp. 303-305. doi:10.1016/j.amc.2003.08.073

[2] A. Y. Ozban, "On the Positive Solutions of the System of Rational Difference Equations $x_{n+1}=\frac{1}{y_{n-k}}$,

$y_{n+1}=\frac{y_{n}}{x_{n-m} y_{n-m-k}}$," Journal of Mathematical Analysis and Applications, Vol. 323, No. 1, 2006, pp. 26-32. doi:10.1016/j.jmaa.2005.10.031

[3] A. Y. Ozban, "On the System of Rational Difference Equations $x_{n}=\frac{a}{y_{n-3}}, \quad y_{n}=\frac{b y_{n-3}}{x_{n-q} y_{n-q}}$, " Applied Mathematics and Computation, Vol. 188, No. 1, 2007, pp. 833837. doi:10.1016/j.amc.2006.10.034

[4] X. Yang, Y. Liu and S. Bai, "On the System of High Or- der Rational Difference Equations $x_{n+1}=\frac{a}{y_{n-p}}$,

$y_{n+1}=\frac{b y_{n-p}}{x_{n-q} y_{n-q}}$, Applied Mathematics and Computation,

Vol. 171, No. 2, 2005, pp. 853-856.

doi:10.1016/j.amc.2005.01.092

[5] G. Papaschinopoulos and C. J. Schinas, "On a System of Two Nonlinear Difference Equations," Journal of Mathematical Analysis and Applications, Vol. 219, No. 2, 1998, pp. 415-426. doi:10.1006/jmaa.1997.5829

[6] G. Papaschinopoulos and B. K. Papadopoulos, "On the Fuzzy Difference Equation $x_{n+1}=A+\frac{x_{n}}{x_{n-m}}$," Fuzzy Sets and Systems, Vol. 129, No. 1, 2002, pp. 73-81. doi:10.1016/S0165-0114(01)00198-1

[7] E. Camouzis and G. Papaschinopoulos, "Global Asymptotic Behavior of Positive Solutions on the System of Rational Difference Equation $x_{n+1}=1+\frac{x_{n}}{y_{n-m}}$,

$y_{n+1}=1+\frac{y_{n}}{X_{n-m}}$," Applied Mathematics and Letters, Vol. 17, No. 6, 2004, pp. 733-737. doi:10.1016/S0893-9659(04)90113-9

[8] Y. Zhang, X. F. Yang, D. J. Evans and C. Zhu, "On the Nonlinear Difference Equation System $x_{n+1}=A+\frac{y_{n-m}}{x_{n}}$, $y_{n+1}=A+\frac{x_{n-m}}{y_{n}}$," Computers and Mathematics with Application, Vol. 53, No. 10, 2007, pp. 1561-1566. doi:10.1016/j.camwa.2006.04.030

[9] E. Camouzis, C. M. Kent, G. Ladas and C. D. Lynd, “On the Global Character of Solutions of the System:

$x_{n+1}=\frac{\alpha_{1}+y_{n}}{x_{n}}, \quad y_{n+1}=\frac{\alpha_{2}+\beta_{2} x_{n}+\gamma_{2} y_{n}}{A_{2}+B_{2} x_{n}+C_{2} y_{n}}$, , Journal of Difference Equations and Applications, Vol. 18, No. 7, 2012, pp. 1205-1252. doi:10.1080/10236198.2011.555406

[10] E. Camouzis, E. Drymonis, G. Ladas and W. Tikjha, "Patterns of Boundedness of the Rational System

$x_{n+1}=\frac{\alpha_{1}}{A_{1}+B_{1} x_{n}+C_{1} y_{n}}, \quad y_{n+1}=\frac{\alpha_{2}+\beta_{2} x_{n}+\gamma_{2} y_{n}}{A_{2}+B_{2} x_{n}+C_{2} y_{n}}$, , Journal of Difference Equations and Applications, Vol. 18, No. 1, 2012, pp. 89-110. doi:10.1080/10236198.2010.515591

[11] H. M. El-Owaidy, A. M. Ahmed and M. S. Mousa, “On Asymptotic Behavior of the Difference

$x_{n+1}=\alpha+\frac{x_{n-k}}{x_{n}}$," Applied Mathematics and Computation, Vol. 147, No. 1, 2004, pp. 163-167. doi:10.1016/S0096-3003(02)00659-8 